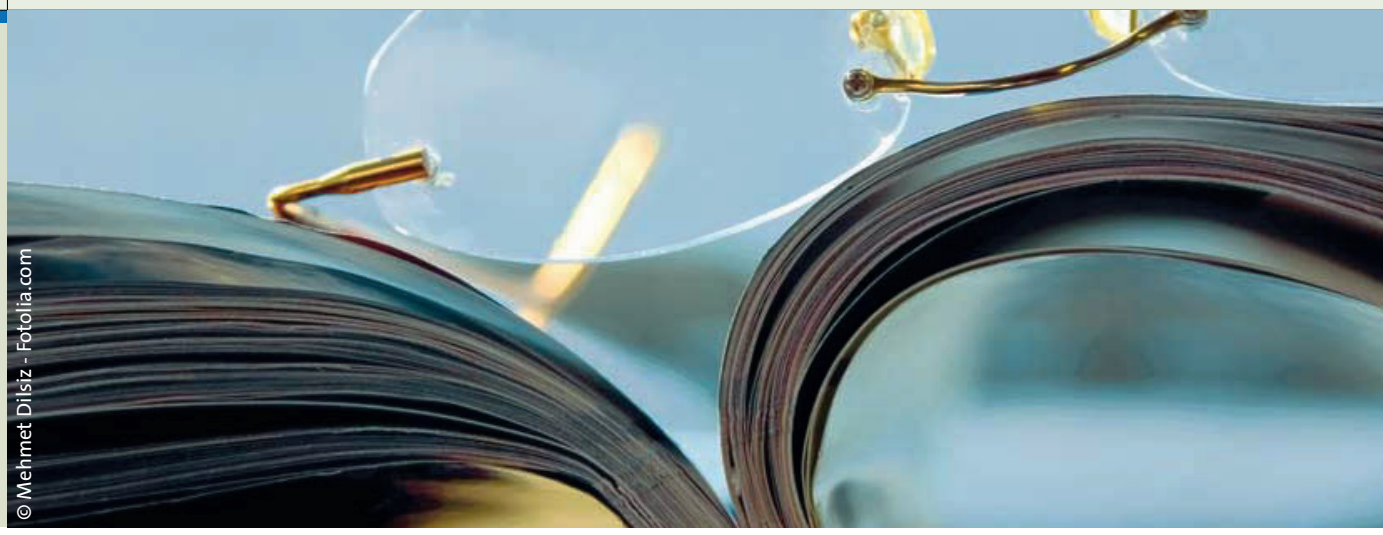

\title{
Allergien: Bauernhof schützt bis ins hohe Alter
}

\author{
Wer auf einem Bauernhof aufgewachsen ist, hat ein deutlich geringeres \\ Allergie-Risiko. Aber wie lange hält dieser Effekt an und in welchem Maß \\ beeinflusst die Urbanisierung das Auftreten von Allergien?
}

nsgesamt 18.087 Jugendliche und Erwachsene kamen in einer schwedischen Populationsstudie der Bitte nach und beantworteten per Internet Fragen zum Vorliegen von allergischen Erkrankungen und möglichen Risikofaktoren. Die Personen stammten zu gleichen Teilen aus der Großstadt Göteborg oder aus anderen Regionen in West-Schweden.

Es wurden vier Altersgruppen gebildet: 16-30, 31-45, 46-60 und 61-75 Jahre. Die Personen, die während der ersten fünf Lebensjahre auf einem Bauernhof gelebt hatten, wiesen ein signifikant geringeres Risiko für eine allergische Rhinitis auf als Personen ohne Kindheit in einem Agrarbetrieb. Dieser Effekt war in der Gruppe der 16- bis 30-Jährigen am stärksten ausgeprägt (19,5 vs. 30,5\%, p<0,001), konnte jedoch auch noch bei Personen zwischen 61 und 75 Jahren nachgewiesen werden ( 16,5 vs. $19,4 \%, p=0,045)$.

Die Analyse zeigte insgesamt eine signifikante Abhängigkeit der Häufigkeit allergischer Rhinitiden von der Einwohnerdichte. Personen in Göteborg und mittel- großen Städten waren häufiger erkrankt als Menschen in Kleinstädten oder ländlichen Gebieten. Allerdings wichen die Prävalenzen hier häufig nur um wenige Prozentzahlen von einander ab und zeigten in der Subgruppe der 61- bis 75-Jährigen keinen signifikanten Unterschied mehr.

Fazit: Nach der vorliegenden Befragungsstudie mit über 18.000 Personen hat der Aufenthalt auf einem Bauernhof im Kleinkindesalter einen protektiven Effekt auf die Entwicklung einer allergischen Rhinitis, der auch noch im hohem Alter messbar ist.

Eriksson J et al. Growing up on a farm leads to lifelong protection against allergic rhinitis. Allergy 2010; May, 17 [Epub ahead of print]

\section{Reduziert Calcitriol Kortikoid-Nebenwirkungen?}

\section{Topische Kortikoide können selbst bei kurzzeitigem Einsatz die epidermale Permeabilität und die antimikrobielle Barrierefunktion beeinträchtigen. Daher untersuchten Forscher aus Korea, ob die gleichzeitige Behandlung mit topischem Calcitriol diese Nebenwirkung mindert.}

$\mathrm{N}$ ach aktuellen Studien könnte Calcitriol, ein Vitamin-D3-Metabolit, der in Keratinozyten synthetisiert wird, eine wichtige Rolle bei der Bildung und Aufrechterhaltung der epidermalen Permeabilität und antimikrobiellen Barriere spielen. Daher überprüfte die Arbeitsgruppe, ob eine topische Behandlung mit Calcitriol ähnliche Effekte erzielt. Sie trug bei haarlosen Mäusen zunächst auf jeder Seite Clobetasol auf und behandelte beide Stellen nach 20 Minuten jeweils mit Calcitriol bzw. einer wirkstofffreien Trägersubstanz. Die- se Prozedur wurde über 3,5 Tage alle zwölf Stunden wiederholt.

24 Stunden nach der letzten Applikation erfassten die Autoren die Permeabilitätsbarrierefunktion und entnahmen Hautproben, die mittels Elektronenmikroskopie sowie Nile-Red-Färbung untersucht wurden. Darüber hinaus wiesen sie anhand von Immunhistochemie, Western Blot und Real-Time ReverseTranskriptase-Polymerase-Kettenreaktion verschiedene körpereigene Substanzen nach, um den zugrunde liegenden Wirkmechanismus darzustellen.
Nach den Ergebnissen ließ sich durch die topische Behandlung mit Calcitriol sowohl die epidermale Permeabilität als auch die antimikrobielle Barrierefunktion verbessern.

Fazit: Aufgrund ihrer Untersuchung gehen die Autoren davon aus, dass die zusätzliche Gabe von Calcitriol zu Kortikoiden in der topischen Therapie tatsächlich die Nebenwirkungen der Kortikoide auf die Haut reduzieren könnte. Darüber hinaus erklären die Daten ihres Erachtens die therapeutischen Effekte des VitaminD-Analogons bei anderen chronischen Dermatosen, für die eine Schädigung der Barrierefunktion charakteristisch ist. pe

Hong SP et al. Topical calcitriol restores the impairment of epidermal permeability and antimicrobial barriers induced by corticosteroids. Br J Dermatol 2010; 162: 1251-6o 